\title{
Top-down Illumination Photometric Stereo Imaging Using Light-Emitting Diodes and a Mobile Device
}

\author{
Emma Le Francois ${ }^{1}$, Johannes Herrnsdorf ${ }^{1}$, Laurence Broadbent ${ }^{2}$, Martin D. Dawson ${ }^{1}$ and Michael J. Strain ${ }^{1}$ \\ ${ }^{1}$ Institute of Photonics, Department of Physics, University of Strathclyde, Glasgow G1 1RD (UK), \\ ${ }^{2}$ Aralia Systems, Bristol Robotics Laboratory, Bristol BS16 1QY (UK) \\ Email: emma.le-francois@strath.ac.uk
}

\begin{abstract}
D reconstruction of objects can be achieved using a top-down illumination, photometric stereo imaging configuration with four modulated white LEDs and a mobile phone. The Standard deviation for the reconstruction is ranging from $3.5 \%$ to $10.4 \%$.
\end{abstract}

(C) 2019 Emma Le Francois

OCIS codes: $100.2000,100.6890$.

\section{Introduction}

Photometric stereo imaging relies on having one fixed camera perspective and different illumination directions to image an object in three dimension (3D) [1]. This technique determines the surface normal vectors and surface albedo at each pixel of the captured frames assuming a perfectly diffuse (Lambertian) surface of the imaged object [1]. Surface normal components can then be integrated to recover the 3D shape. So far, the most common photometric stereo configuration is as implemented with: a commercial camera placed in front of the object and, at least, four white light-emitting diodes (LEDs) surrounding it in a top/bottom/left/right or X shape [2,3]. A fast 3D reconstruction has been reported with white LEDs surrounding a camera, where LEDs were sequentially lit by a USB programmable board [2]. These 3D reconstructions showed a standard deviation error ranging from $2.65 \mathrm{~mm}$ to $15.60 \mathrm{~mm}$ for objects of size $50 \mathrm{~mm}$ and $160 \mathrm{~mm}$, respectively [2].

In order to unlock the potential use of LEDs in industrial or public spaces for monitoring, security check or 3D imaging on mobile devices, the combination of lighting/camera system has to be easy to set up and flicker free. LEDs are affordable, energy efficient and have a fast modulation bandwidth ranging from several $\mathrm{MHz}$ up to $\mathrm{GHz}$ [4]. Thanks to a convenient interfacing with digital electronics [5], LED lighting enables advanced functionality such as wireless optical networking through the illumination itself.

Here we report the 3D reconstruction of objects using a top-down illumination photometric stereo imaging configuration. Four white modulated LEDs, mounted on a ceiling, illuminate the object while a mobile phone captures frames at 960 frame per second (fps). The LEDs are modulated at the camera frame rate with orthogonal multiple access carriers such that visible flicker is minimal and no electronic synchronization is needed between the LEDs and the phone. The 3D reconstruction shows a root mean square error (RMSE) ranging from 3.5\% to $10.4 \%$ depending on the complexity of the object.

\section{Experiment}

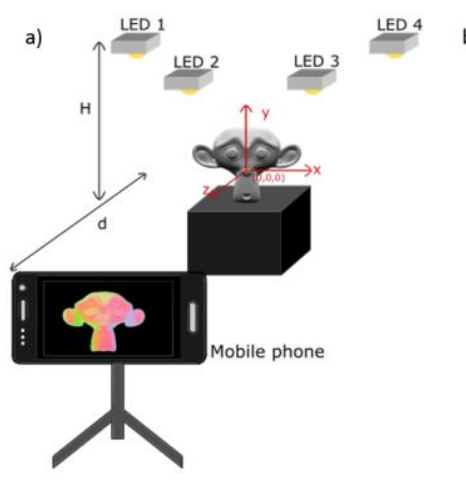

b)
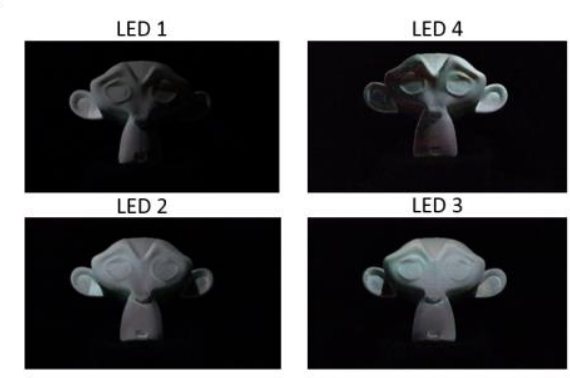

Fig. 1. a) Schematic of the experimental setup, b) Four images obtained from each fours LEDs after decoding

As illustrated in Fig. 1.a), four white LEDs (Osram OSTAR Stage LE RTUDW S2W) were placed on a gantry above the object at a height of $\mathrm{H}=46 \mathrm{~cm}$. In our experiments we used a number of 3D printed objects, including geometric solids and more complex shapes such as the monkey head in Fig. 1. The objects were $\sim 130 \mathrm{x} 94.5 \mathrm{~mm}$ wide and $79 \mathrm{~mm}$ deep, with the reference $(0,0,0)$ point taken as their geometric centre. A mobile phone device 
(Samsung Galaxy 9) was mounted on a tripod in front of the object on the $\mathrm{z}$ axis at a distance $\mathrm{d}=30 \mathrm{~cm}$ with a field of view of 59 degrees. The phone was in a "side acquisition" configuration. The phone captured frames with a resolution of $1280 \times 720$ at a rate of 960 frame per second (fps) for 0.2 s. Fig. 1.b) shows the four images obtained after decoding the frames. For the illumination, we used an USB programmable controller board (Arduino Uno) to modulate the four LEDs at a frequency of $960 \mathrm{~Hz}$. Each LED was modulated with an individual multiple access carrier signal at a frequency of $960 \mathrm{~Hz}$, which is above visual flicker recognition and therefore suitable for digital lighting applications. The carriers were designed such that, analogous to orthogonal frequency division multiple access, no synchronization between the LEDs and the mobile phone was required.

\section{Results}
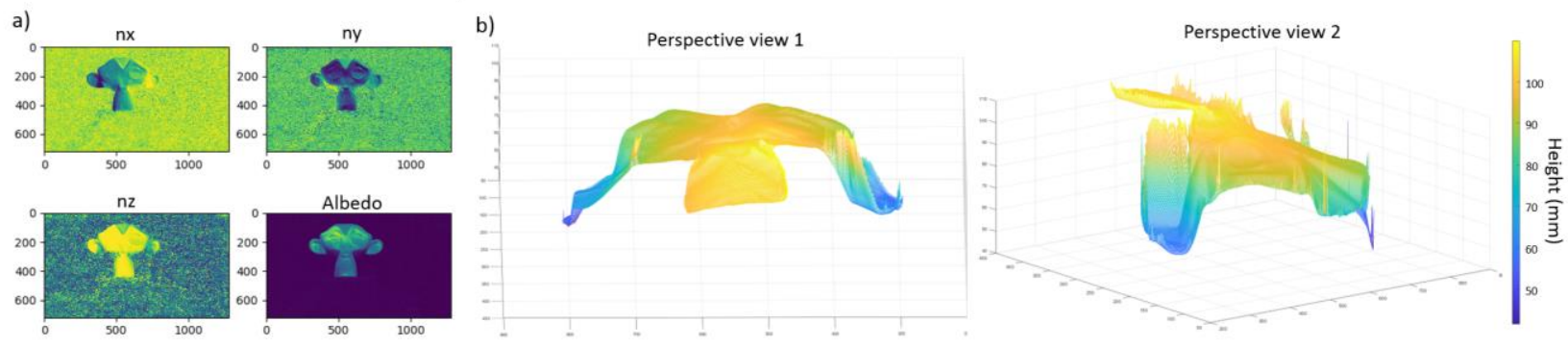

Fig. 2. a) Components of the surface normal vectors and the albedo, b) Different views of the monkey head 3D reconstruction

Surface normal integration has been a challenge for years in computer vision [6]. In this work, we integrate the surface normal vectors with the Fast Marching method [7] to take advantage of its reconstruction speed. A representative set of the surface normal components $n_{x}, n_{y}, n_{z}$, and the surface albedo are shown in Fig. 2.a). Yellow and blue color respectively represent positive and negative value of the surface normal components. As expected from the scheme in Fig. 1.a), $\mathrm{n}_{\mathrm{x}}$ correctly distinguishes left and right facing surfaces of the object. Similarly, $\mathrm{n}_{\mathrm{y}}$ correctly identifies up and down facing surfaces (clearly visible around the nose region), though we generally observe a poorer fidelity as compared to $\mathrm{n}_{\mathrm{x}}$ due to the top-down illumination configuration. Finally, as we cannot see the back of the object, $\mathrm{n}_{\mathrm{z}}$ is always positive with some variations due to the depth of the object.

Despite errors in $\mathrm{n}_{\mathrm{x}}, \mathrm{n}_{\mathrm{y}}$ and $\mathrm{n}_{\mathrm{z}}$, especially in the ear area and the bottom of the face, 3D results plotted in Fig. 2.b) are comparable to the work done in [2]. As a reference, for a $48 \mathrm{~mm}$ diameter sphere and a $75 \mathrm{~mm}$ cube, in a similar configuration, we obtained a RMSE of respectively $5 \mathrm{~mm}$ and $2.6 \mathrm{~mm}$ which corresponds to the same RMSE range as [2]. The normalized RMSE of both objects equals to $10.4 \%$ and $3.5 \%$, respectively.

\section{Conclusion}

Accurate 3D reconstruction is possible in a new photometric stereo configuration that can readily be employed in conventional room lighting scenarios. Top-down illumination photometric stereo can be directly applied to digital lighting application in public areas or industrial applications in the near future. Furthermore, we demonstrated that this method can then be implemented using commercially available, handheld mobile devices. Importantly, operation above the visual flicker recognition threshold is possible and there is no requirement for synchronization between LEDs, thus significantly simplifying installation and deployment.

\section{References}

[1] R. J. Woodham, "Photometric Method For Determining Surface Orientation From Multiple Images,” Opt. Eng., vol. 19, no. 1, pp. 139-144, 1980.

[2] Y. Zhang, G. M. Gibson, R. Hay, R. W. Bowman, M. J. Padgett, and M. P. Edgar, “A fast 3D reconstruction system with a low-cost camera accessory," Sci. Rep., vol. 5, pp. 1-7, 2015.

[3] J. Herrnsdorf, L. Broadbent, G. C. Wright, M. D. Dawson, and M. J. Strain, "Video-Rate Photometric Stereo-Imaging with General Lighting Luminaires," pp. 483-484, 2017.

[4] J. Grubor, S. Randel, K. Langer, and J. W. Walewski, "Broadband Information Broadcasting Using LED-Based Interior Lighting," vol. 26, no. 24, pp. 3883-3892, 2008.

[5] J. J. D. McKendry et al., "Individually addressable AlInGaN micro-LED arrays with CMOS control and subnanosecond output pulses," IEEE Photonics Technol. Lett., vol. 21, no. 12, pp. 811-813, 2009.

[6] Y. Quéau, J. D. Durou, and J. F. Aujol, "Normal Integration: A Survey,” J. Math. Imaging Vis., vol. 60, no. 4, pp. 576-593, 2018.

[7] J. Ho, J. Lim, M.-H. Yang, and D. Kriegman, "Integrating Surface Normal Vectors Using Fast Marching Method,” Comput. Vision-ECCV 2006, pp. 239-250, 2006. 